\title{
Expression profiling of endogenous secretory receptor for advanced glycation end products in human organs
}

Chunmei Cheng ${ }^{1,2}$, Koichi Tsuneyama ${ }^{1,2}$, Rieko Kominami ${ }^{3}$ Harumichi Shinohara $^{3}$, Shigeru Sakurai ${ }^{4}$, Hideto Yonekura ${ }^{4}$, Takuo Watanabe ${ }^{4}$, Yasuo Takano ${ }^{1}$, Hiroshi Yamamoto ${ }^{4}$ and Yasuhiko Yamamoto ${ }^{4}$

${ }^{1}$ Department of Pathology, Toyama Medical and Pharmaceutical University, Toyama, Japan; ${ }^{2}$ Department of Pathology, 21st COE Program, Toyama Medical and Pharmaceutical University, Toyama, Japan; ${ }^{3}$ Department of Anatomy, Kanazawa Medical University, Ishikawa, Japan and ${ }^{4}$ Department of Biochemistry and Molecular Vascular Biology, Kanazawa University Graduate School of Medical Science, Kanazawa, Japan

\begin{abstract}
The receptor for advanced glycation end products (RAGE) is a cell surface multiligand receptor of the immunoglobulin superfamily, which participates in physiological and pathological processes such as neuronal development, diabetes, inflammation, neurodegenerative disorders, and cancer. A novel splice variant of RAGE-endogenous secretory decoy form (esRAGE) was recently identified and is thought to be a prospective candidate to modify these RAGE-associated conditions. Here, we investigated the expression and distribution of esRAGE and RAGE proteins with domain-specific antibodies. We studied a wide variety of adult normal human preparations obtained from surgical and autopsy specimens using a tissue microarray technique. The results revealed that esRAGE was widely distributed and we classified its expression into four patterns. In pattern $A$, the cytoplasm is stained diffusely in neurons, vascular endothelium, pneumocytes, mesothelium, pancreatic $\beta$ cells, and macrophages/monocytes. In pattern B, dot-like granules are stained in the supranuclear regions facing the luminal surface of the bile ducts, salivary glands, digestive tracts, renal tubules, prostate, skin, thyroid, and bronchioles. Pattern $\mathrm{C}$ is represented by diffuse staining in the stromal area of the arterial walls. Pattern D shows diffuse and strong staining of secreted materials such as thyroidal colloid, crystals in renal tubular lumen, and glandular lumen in prostate. This study provides, for the first time, a histopathological basis for understanding the physiological roles of esRAGE in humans, and will contribute to elucidating the participation of esRAGE in pathological processes and to exploring novel diagnostic and therapeutic concepts. Modern Pathology (2005) 18, 1385-1396. doi:10.1038/modpathol.3800450; published online 3 June 2005
\end{abstract}

Keywords: receptor for advanced glycation end products (RAGE); endogenous secretory RAGE (esRAGE); immunohistochemistry; normal human organs

The receptor for advanced glycation end products (RAGE) is a multiligand member of the immunoglobulin superfamily of cell surface molecules, whose extracellular region consists of one V-type and two C-type immunogloblin-like domains. ${ }^{1,2}$ The $\mathrm{V}$ domain is critical for the binding of distinct ligands, which have been implicated in development, main-

Correspondence: Dr K Tsuneyama, MD, PhD, Department of Pathology, 21st COE Program, Toyama Medical and Pharmaceutical University, 2630 Sugitani, Toyama 930-0194, Japan and Dr Y Yamamoto, MD, PhD, Department of Biochemistry and Molecular Vascular Biology, Kanazawa University Graduate School of Medical Science, 13-1 Takara-machi, Kanazawa 920-8640, Japan. E-mails: ktsune@ms.toyama-mpu.ac.jp and yasuyama@med. kanazawa-u.ac.jp

Received 6 February 2005; revised and accepted 18 April 2005; published online 3 June 2005 tenance of tissue homeostasis, proinflammatory reactions, and diseases such as diabetes, neurodegenerative disorders, and cancer. ${ }^{1}$ Yonekura et al identified novel splice variants of RAGE mRNA coding for a N-terminally truncated, membranebound form and a C-terminally truncated secretory form, which they designated endogenous secretory RAGE (esRAGE), as well as the known full-length membrane-bound form. The N-terminally truncated form lacked the ability to bind ligands such as AGE and had much lower expression due to deletion of signal sequence and N-glycosylation sites, whereas esRAGE was efficiently secreted from cultured cells, and was capable of capturing the ligands and thought to work as a decoy-type receptor. ${ }^{3}$

Since the ligand-RAGE interaction is considered a potential therapeutic target of relevant diseases, it 
is important to gain knowledge about the esRAGE distribution in normal human organs and tissues. To this end, we conducted this study to examine the expression of esRAGE as well as the full-length and $\mathrm{N}$-terminally truncated isoforms in a wide repertoire of surgical and autopsy specimens, registered in Toyama Medical and Pharmaceutical University, using a tissue microarray technique with RAGE domain-specific antibodies. The immunohistochemical analysis revealed at least four distinct patterns of esRAGE expression in different human organs, which would reflect different functional roles in respective organs.

\section{Materials and methods}

\section{Organs and Tissues}

From surgical and autopsy specimens registered in Toyama Medical and Pharmaceutical University, formalin-fixed, paraffin-embedded tissue blocks of disease-free organs were subjected to this study; they included brain, thyroid, salivary glands, heart, aorta, lung with bronchi, liver, pancreas, spleen, esophagus, stomach, small intestine, colon, prostate, and skin. In all, 12 cases per organ were randomly selected, and set in one tissue microarray block as described previously. ${ }^{4}$ The blocks were cut into $4-\mu$ m-thick sections and processed for immunohistochemistry as well as hematoxylin and eosin (H\&E) staining.

\section{H\&E Staining}

The sections from all tissue microarray blocks first underwent routine H\&E staining to enable histopathological review, and were proven free of disease.

\section{Primary Antibodies and Immunohistochemistry}

The antibodies and RAGE domains observed are schematically represented in Figure 1; the goat polyclonal antibody against the V domain of RAGE proteins (Chemicon, CA, USA; dilution, 1:800), recognizes the full-length RAGE and esRAGE; the rabbit polyclonal antibody against the C-terminal 16 amino acids of esRAGE (dilution, 1:1000) recognizes only esRAGE; ${ }^{3}$ and the rabbit polyclonal antibody against the C-terminal intracellular domain of RAGE (C-terminal 20 amino acids, Santa Cruz Biotech., CA, USA; dilution, 1:100) recognizes the full-length and N-terminally truncated RAGE proteins. Immunostaining procedures were based on a new microwave technique as described previously. ${ }^{5}$ In brief, the sections were deparaffinized, dehydrated, and treated for antigen retrieval with TRS-buffer solution (TRS; DAKO, CA, USA) in a wet chamber in a microwave oven (Type RE-11, Sharp, Tokyo, Japan; maximum $500 \mathrm{~W}$ ) for $15 \mathrm{~min}$. The sections were

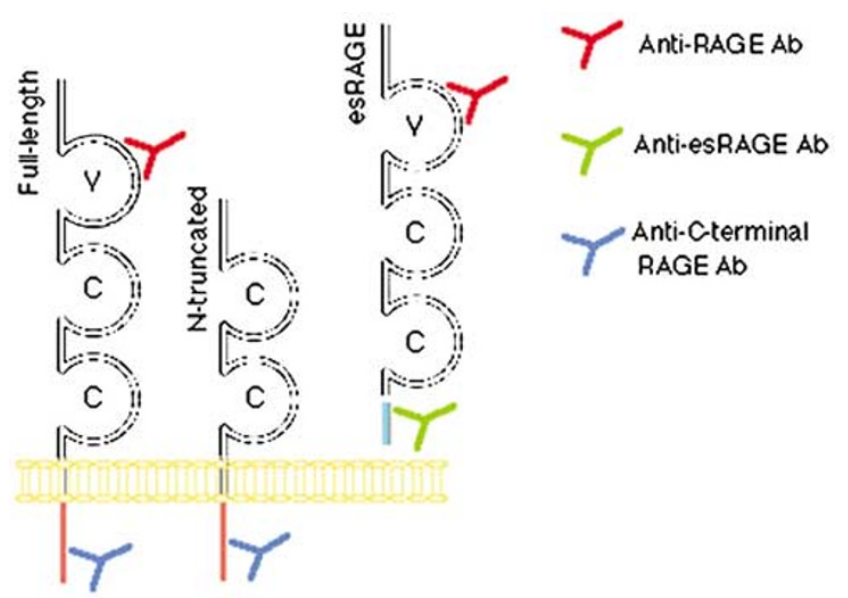

Figure 1 Structures of full-length RAGE, N-terminally truncated RAGE and esRAGE, and antibodies used in this experiment. The goat polyclonal anti-RAGE antibody (Chemicon) can recognize $\mathrm{V}$ domain of both full-length RAGE and esRAGE, while the rabbit polyclonal anti-esRAGE antibody is specific to esRAGE. The rabbit polyclonal anti-C-terminal RAGE antibody can recognize full-length RAGE and N-terminally truncated RAGE, but not esRAGE.

allowed to cool at room temperature for $30 \mathrm{~min}$, rinsed under running water for $1 \mathrm{~min}$, and then sequentially treated with $3 \% \mathrm{H}_{2} \mathrm{O}_{2}$ for $5 \mathrm{~min}$ to exhaust endogenous peroxidase. After washing with Tris-buffered saline containing $0.1 \%$ Tween-20 (TBS-T) and blocking with $5 \%$ normal bovine serum albumin (Sigma, USA) for $1 \mathrm{~min}$, the sections were incubated with a primary antibody in a wet chamber, with intermittent microwave irradiation (MI-77, Azumaya, Japan) for $10 \mathrm{~min}$. After washing with TBS-T, the peroxidase-labeled polymer for rabbit polyclonal antibody (EnVision, DAKO, Carpinteria, CA, USA) and goat polyclonal antibody (Histofine, Simple Stain, NICHIREI, Tokyo, Japan) were applied for $10 \mathrm{~min}$ in a wet chamber with intermittent microwave irradiation. After washing with TBS, color was developed with 3-3'-diaminobenzidine (DAB) (Sigma, USA). The sections were then counterstained with Meyer's hematoxylin, and cover-slipped for microscopic observation. Brown areas were judged to be positive based on the manufacturer's information (Envision System).

\section{Absorption Test}

The absorption test was performed to prove the specificity of the immunostaining. In brief, an excess volume of recombinant esRAGE protein was added to diluted anti-esRAGE polyclonal antibody and mixed well using a Vortex. This preabsorption reagent was kept at room temperature for more than $1 \mathrm{~h}$. Selected serial sections were stained with nonpreabsorbed and preabsorbed antibodies using the regular staining procedures. Staining for esRAGE 
was judged positive when the signal decreased or disappeared with the preabsorbed antibody compared with the non-preabsorbed antibody.

\section{Double Immunostaining}

To further define the characteristics of esRAGE expression in various organs, cytomarkers of target cells were employed to perform double immunostaining; they included bile canalicular marker CD10, cell proliferative marker MIB-1, Langerhans' islet A, B, D, and PP cell markers glucagon, insulin, somatostatin and pancreatic polypetide (PP), and heparan sulfate proteoglycans (HSPG), essential components of the extracellular matrix and basement membrane. The primary antibodies used were rabbit polyclonal antibodies against human glucagon, insulin, and somatostatin (DAKO, Carpinteria, CA, USA; prediluted), against PP (ibid. dilution, 1:900), and against HSPG (gift from Dr T Saku, Niigata University), and mouse monoclonal antibodies against CD10 (Novocastra, UK; dilution, 1:40) and against Ki-67 (MIB-1) (DAKO, Carpinteria, CA, USA; dilution, 1:50). For esRAGE and glucogon, esRAGE and insulin, esRAGE and somatostatin, esRAGE and PP, esRAGE and MIB-1, and esRAGE and HSPG double staining, esRAGE was first immunoreacted and visualized with DAB (Sigma, USA), and the latter antigens were then visualized with Fast Blue (Vector). In the case of CD10 and esRAGE double staining, CD10 was first immunostained with DAB, and then esRAGE was immunostained with Fast Blue. In double immunostaining, hematoxylin counterstaining was omitted. To avoid interference due to recognition of the primary antibody in the secondary antibody application step, the specimens were soaked in boiled water for $15 \mathrm{~min}$ to denature the applied proteins. ${ }^{6}$ This treatment was confirmed to result in negative staining with peroxidase-conjugated secondary antibodies against mouse or rabbit IgG. Loci colored brown and/or blue were thus evaluated as positive.

\section{Results}

To examine esRAGE expression in each organ, tissue microarray specimens were displayed as 12 independent cases per slide with each case being in $5 \mathrm{~mm}$ in diameter (Figure 2). The tissue microarray specimens were counterstained with $\mathrm{H} \& \mathrm{E}$ and then probed with antibody specific to human esRAGE. All samples were independently assessed by plural analysts (CC and KT), and the resultant coordinated evaluations are presented. When the scores determined by the two pathologies differed from each other, a consensus score was derived by a joint examination.

esRAGE staining showed four distinctive types of distribution, which we tentatively designated patterns A, B, C and D.
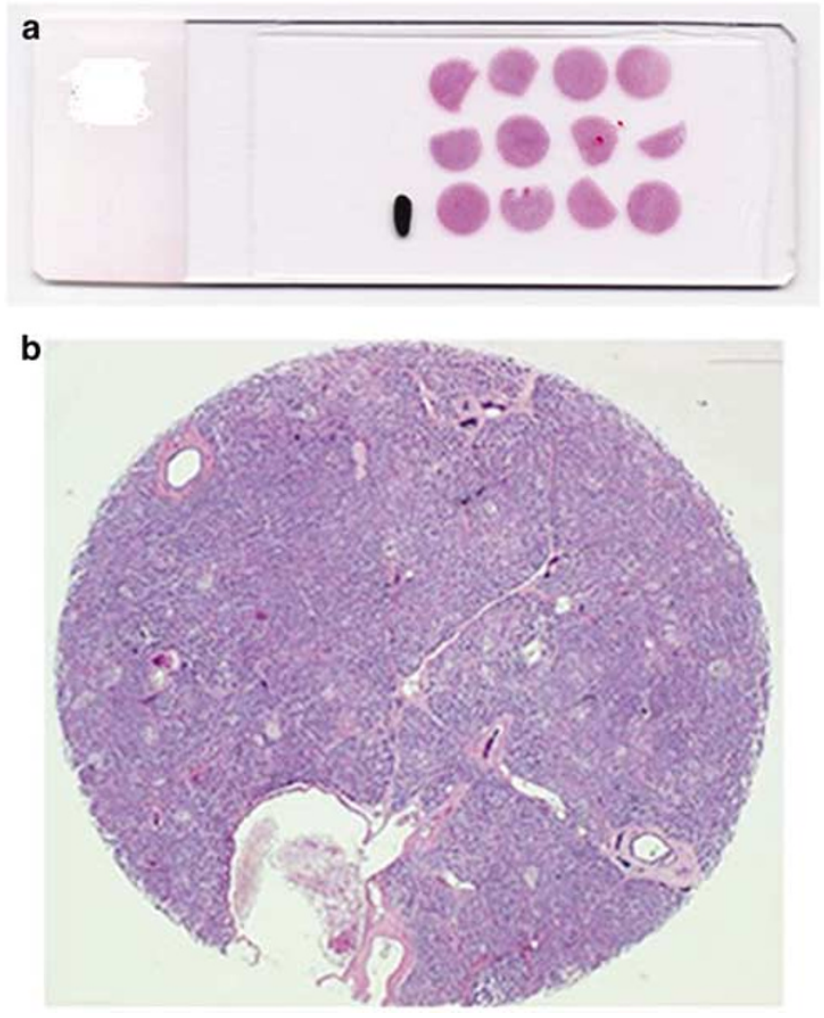

Figure 2 Tissue microarray specimen with different 12 cases in one slide was prepared for each organ examined. (a) Each array sample ( $5 \mathrm{~mm}$ in diameter) was excised from the original block and re-embedded in paraffin. This tissue microarray technique is useful for comparing the staining pattern with no technical variations. (b) One array sample (pancreas) of 5-mm diameter enables almost the same visualization as a visual field of $\times 40$ magnification. Their content of array samples is sufficient for pathological observation.

Pattern A was characterized by diffuse cytoplasmic staining. As shown in Figure 3, this pattern was exemplified by the following cell types: the cell body of neurons in the cerebellum and cerebrum, mesothelial cells and cardiac muscle cells of the heart, vascular endothelial cells (EC), type II pneumocytes, macrophages/monocytes in the alveolar lumen, cells in islets of Langerhans, and medullary cells of the adrenal gland; parts of the pancreatic acinus also showed this pattern but with a weaker signal. Double immunostaining revealed that esRAGE reactivity was colocalized with insulin reactivity in the islets of Langerhans, as evidenced by the color produced when brown (esRAGE) and blue (insulin) were merged (Figure 4).

Pattern B was characterized by supranuclear dotlike granular staining that faced to the luminal surface of the epithelium (Figure 5). This pattern was marked in epithelia of bile ducts, acinus and part of ductal epithelia of the salivary gland, basal cells of the squamous epithelia of the esophagus, superficial-foveolar epithelia and gland of the digestive tract, renal tubular epithelia of the kidney, 

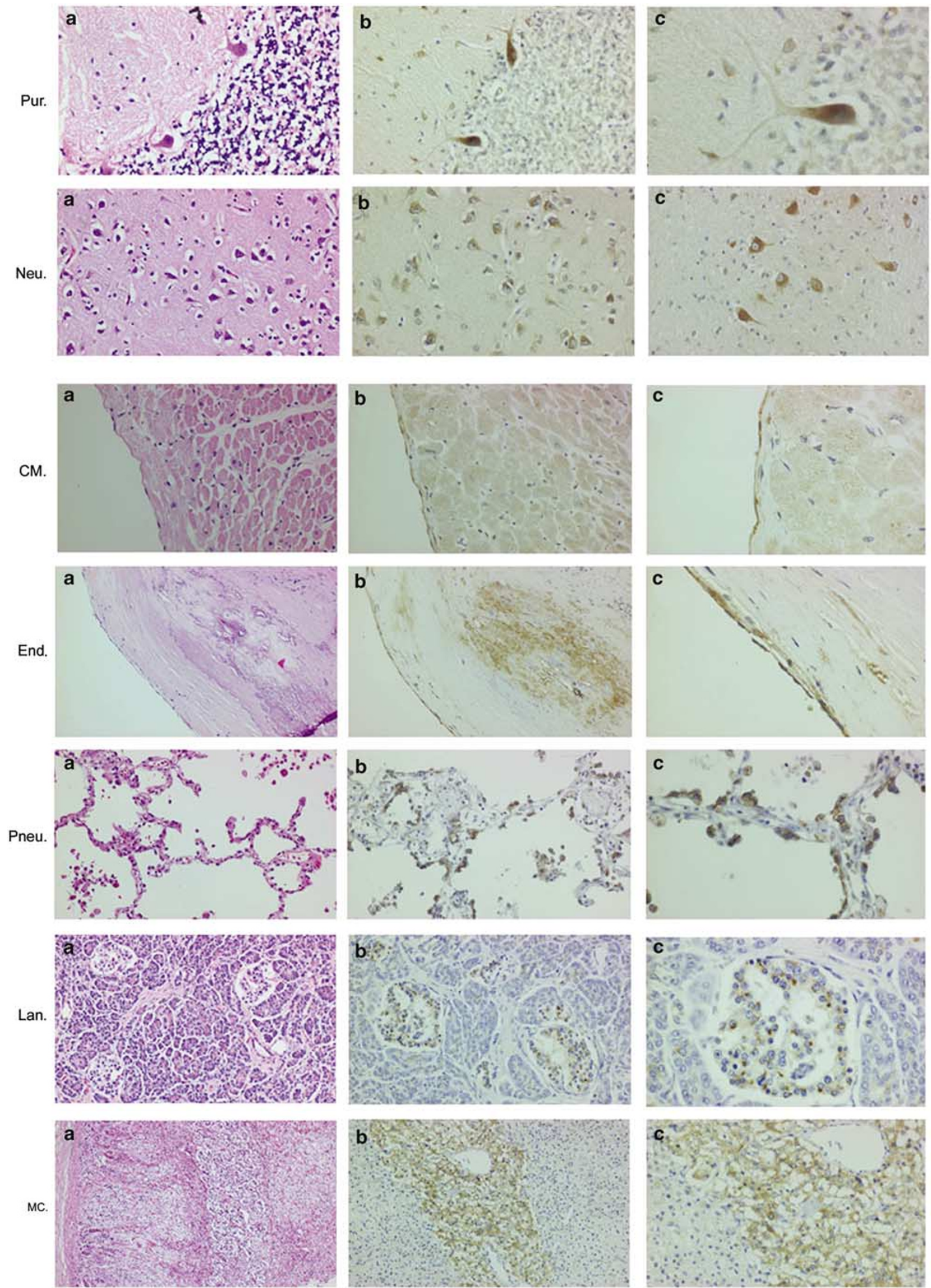

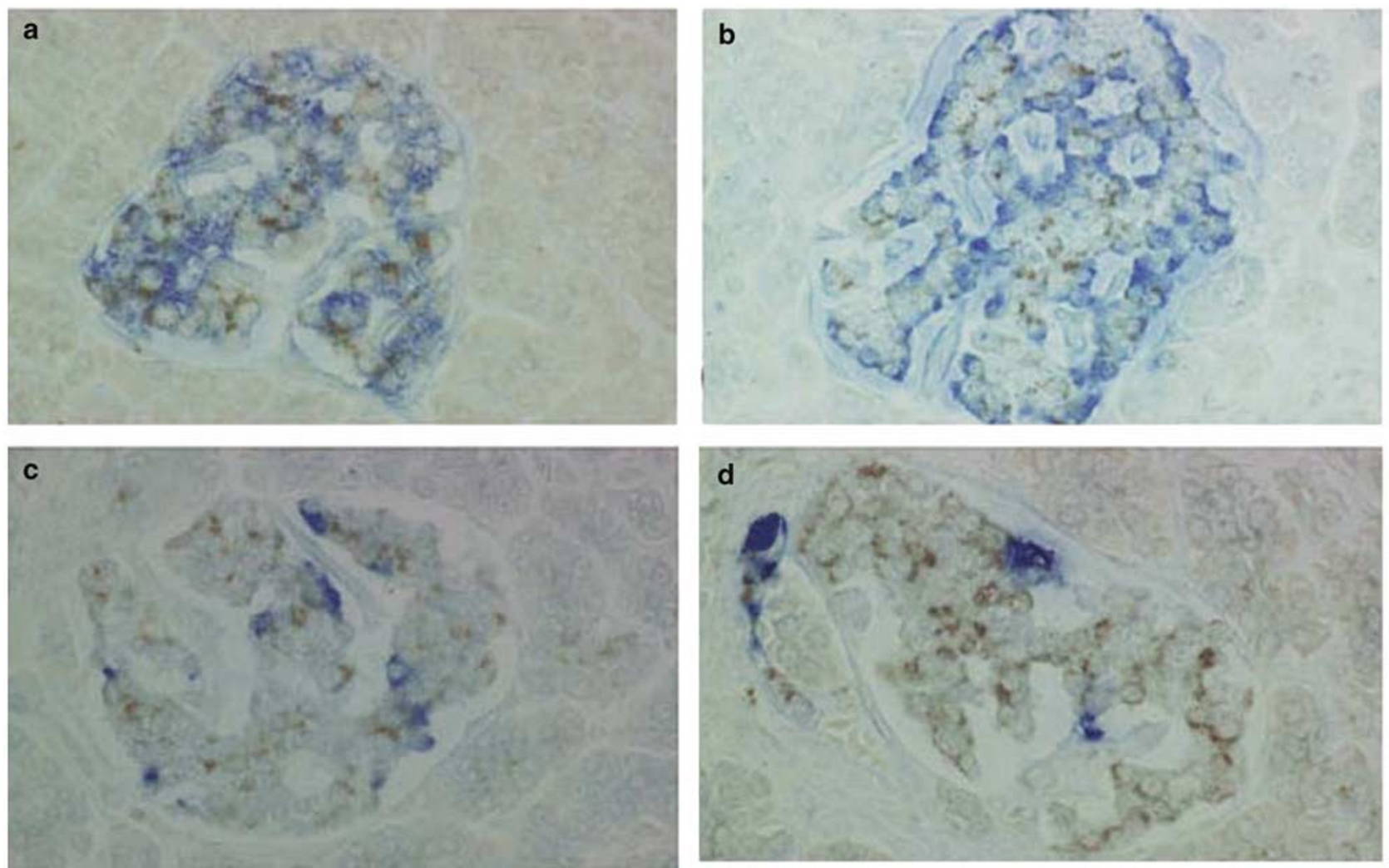

Figure 4 Double immunostaining for esRAGE and (a) insulin, (b) glucagon, (c) somatostatin, or (d) pancreatic polypeptide in pancreatic islet cells. esRAGE expression was detected as brown, sharing an insulin area in blue (a), with no areas sharing glucogon, somatostatin, and pancreatic polypeptide in blue (b-d).

glandular epithelia of the prostate, basal cells of the squamous epithelia of epidermis, and ductal epithelia of the accessory gland of the skin. Follicular epithelia of the thyroid and glandular epithelia of the bronchioli also exhibited this pattern, but with a weak signal (data not shown). Double immunostaining of hepatocytes revealed that esRAGE was colocalized with CD10 along the line of the bile canaliculi (Figures 6a and b).

Diffuse staining in the stromal area was designated as pattern C. As shown in Figure 7, this pattern was marked in the wall of the coronary artery and aorta with mild atherosclerosis. Double immunostaining identified HSPG as a biomarker colocalized at the same loci as esRAGE.

Pattern D featured diffuse and strong staining in the secreted materials. As shown in Figure 8, this was marked in colloid of the thyroid, crystals in the renal tubular lumen, and secreted materials in glandular lumen of the prostate.
All esRAGE staining described above was completely abolished after preabsorption with human esRAGE protein (Figure 9), and thus were judged to represent specific recognition by the antibody.

When the same tissue microarray specimens were probed with a goat polyclonal antibody against the extracellular V domain of human RAGE, the staining patterns were similar to those obtained with the esRAGE-specific antibody, except for minor variations. Typical staining in the brain, liver, and lung is shown in Figure 10. Among them, somewhat distinct immunostaining was noted in hepatocytes; the rabbit anti-esRAGE antibody gave dot-like cytoplasmic staining, while the goat anti-extracellular domain antibody gave diffuse cytoplasmic staining. On the other hand, positive staining with a rabbit polyclonal antibody against the carboxyterminal cytoplasmic domain of human RAGE was restricted to only a few specimens, such as

Figure 3 Cells from tissues and organs represented diffuse cytoplasmic staining (A pattern) with the esRAGE-specific antibody. Three figures are shown for one tissue or organ. (a) HE staining at lower magnification. (b) Staining for esRAGE at lower magnification. (c) Staining for esRAGE at higher magnification. Pur., Purkinje cells of cerebellar cortex; Neu., neurons of cerebrum; CM, cardiac muscle with mesothelial cells; End., endothelial cells of small vessels; Pneu., part of pneumocytes; Lan., Langerhans' islet of the pancreas; MC., medullary cells of adrenal gland. 
Hep.

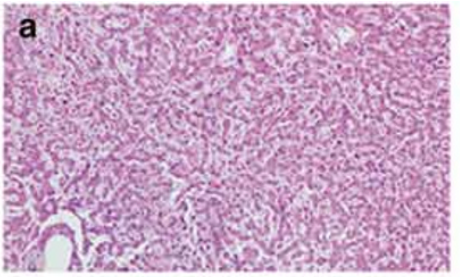

SG.

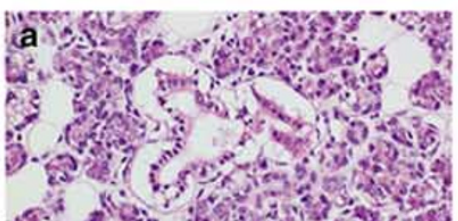

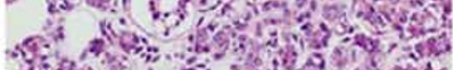
Hon a d

Eso.

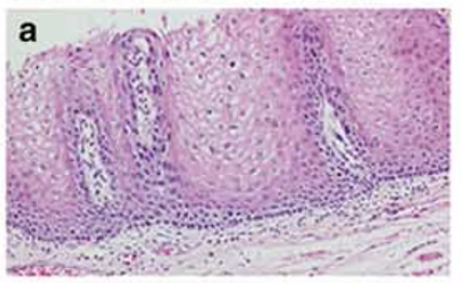

Sto.

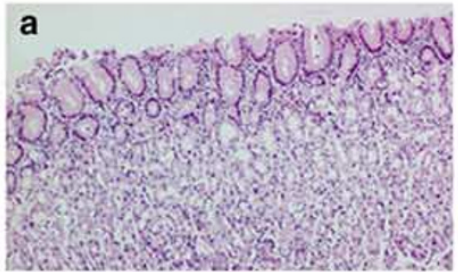

Kid.
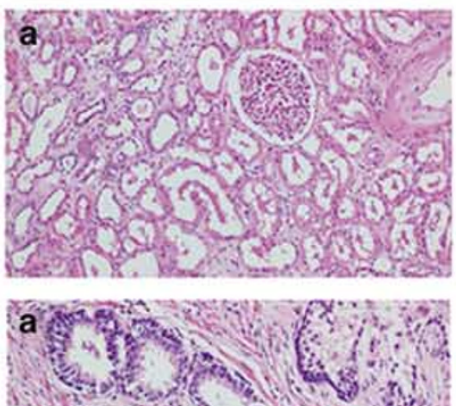

Prost.
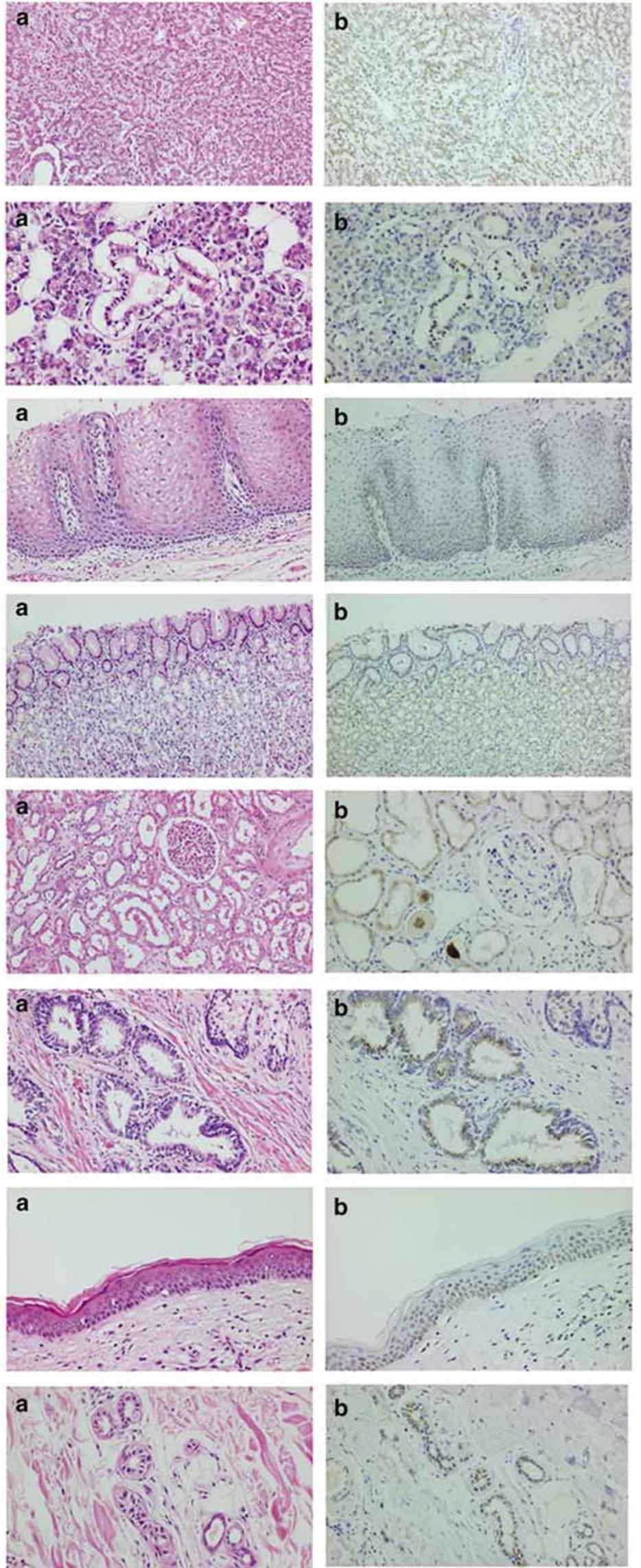
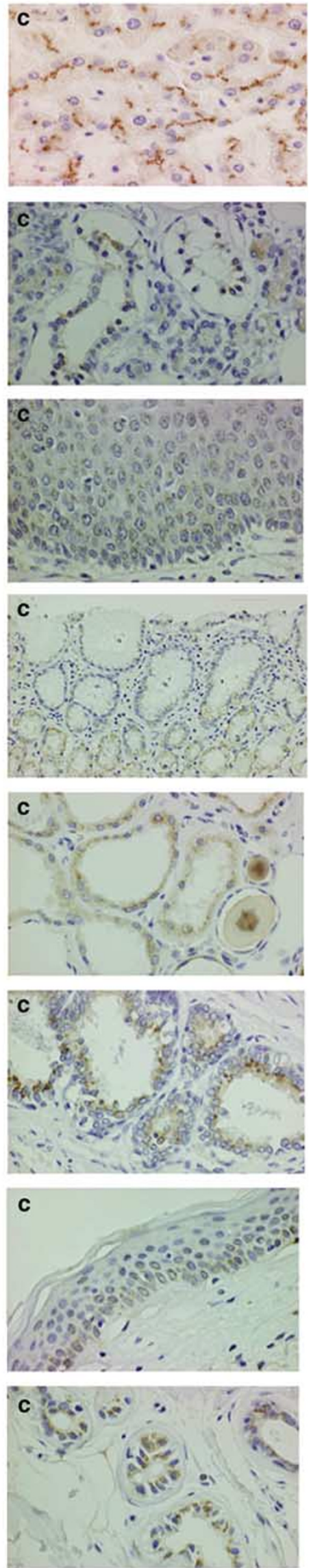

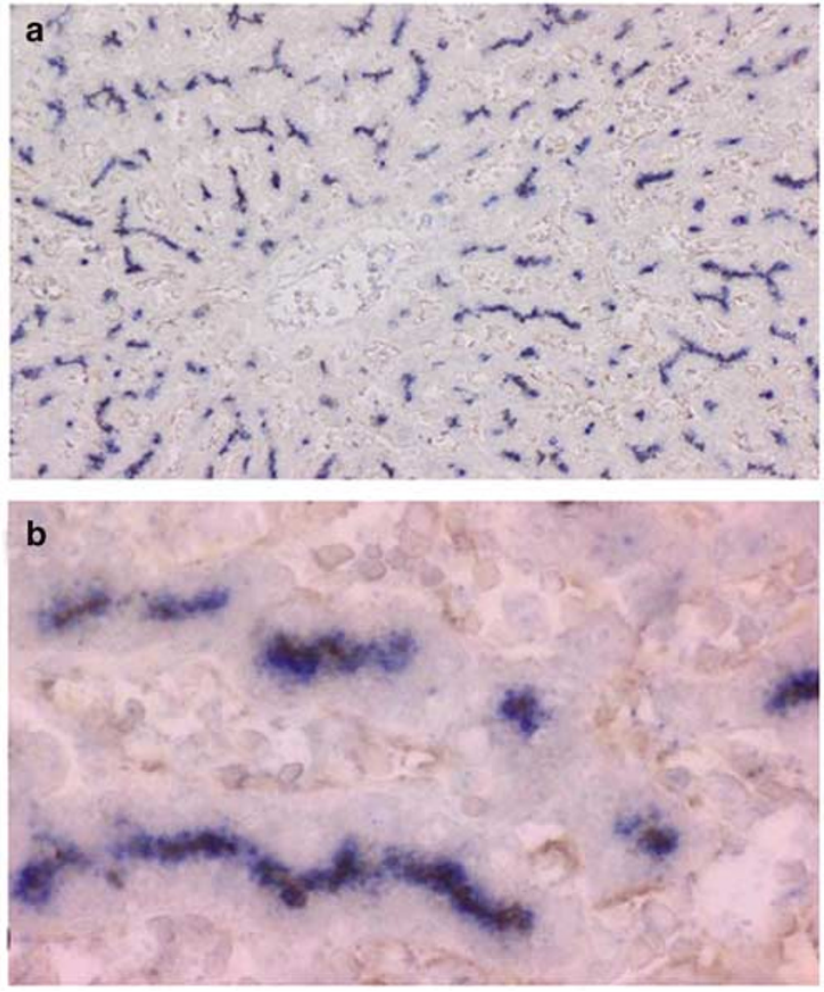

Figure 6 Double immunostaining for esRAGE and CD10, a marker of bile canaliculi. esRAGE and CD10 were colored in blue and brown, respectively. Lower (a) and higher (b) magnifications exhibit dot-like esRAGE expression along the line of CD10positive bile canaliculi.

regenerated type II pneumocytes and part of the pancreatic islet cells (Figure 11).

Tables 1 and 2 summarize the data of esRAGE expression.

\section{Discussion}

The present study was conducted as the first systemic analysis of esRAGE expression in human tissues and organs. The method employed was the tissue microarray technique., ${ }^{4,5}$ This immunostaining method ensures a reproducible comparison among a variety of tissues from one individual or the same tissues from different individuals, which were aligned on the same slide. Tissue or cellular staining for esRAGE was classified into four different patterns, which might reflect distinct functional roles of this decoy receptor in different organs.
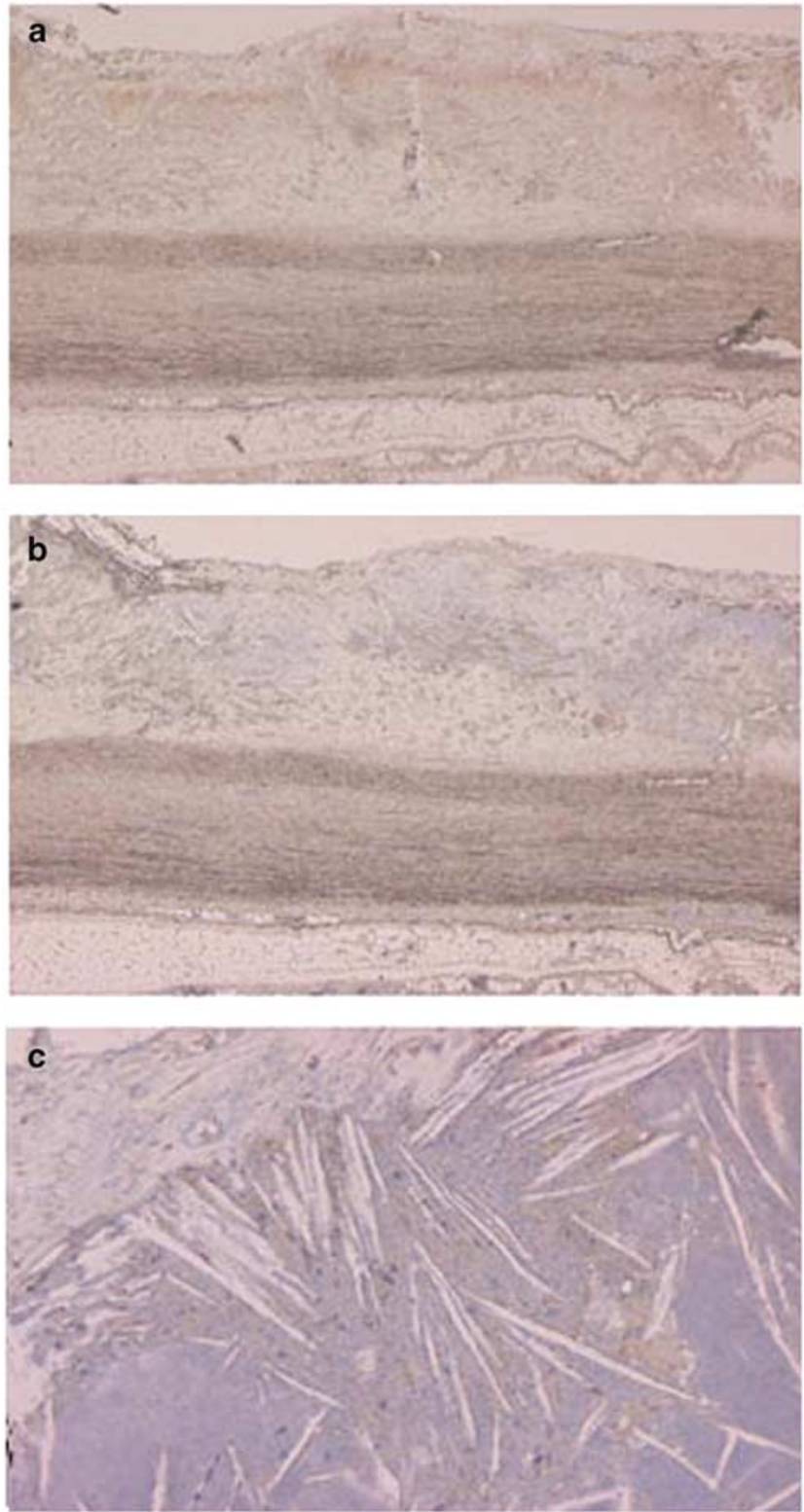

Figure 7 Diffuse staining of esRAGE in the stromal area (pattern C) in the vessel wall (a). Expression of esRAGE in brown and HSPG (perlecan) in blue was identified in the atheromatous area of the intima by double immunostaining at lower (b) and higher (c) magnification.

Pattern A, diffuse cytoplasmic staining, may represent esRAGE in the secretory pathway, because the same areas of Langerhans' islets were positively stained for both insulin and esRAGE (Figure 4). Coexistence of the secretory granules of esRAGE and

Figure 5 The esRAGE-expressing cells from tissues and organs with dot-like supranuclear expression mainly facing to the luminal surface (B pattern). Three figures are shown for one tissue or organ. (a) HE staining at lower magnification. (b) Staining for esRAGE at lower magnification. (c) Staining for esRAGE at higher magnification. Hep., hepatocyte of the liver; SG, salivary gland acinus and duct epithelia; Eso., basal cells of esophagus; Sto., foveolar epithelia and gland of the stomach; Kid., renal tubular epithelia; Prost., glandular epithelia of the prostate; Epi., epidermal basal cells; SA, ductal epithelia of skin appendage. 

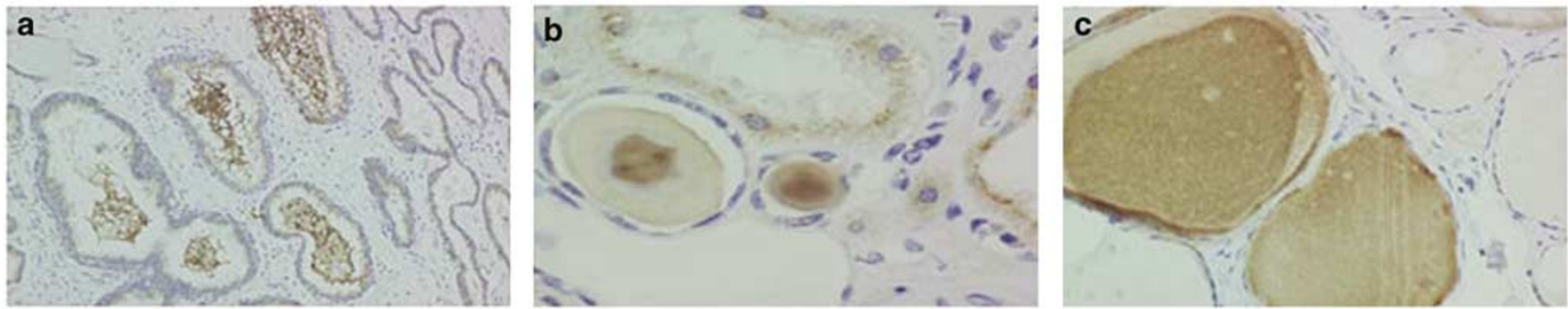

Figure 8 Strong esRAGE expression in secreted materials of the prostatic gland (a), crystals in the renal tubular lumen (b), and colloids of the thyroid follicle (c). This pattern was classified as pattern D.

Lan.
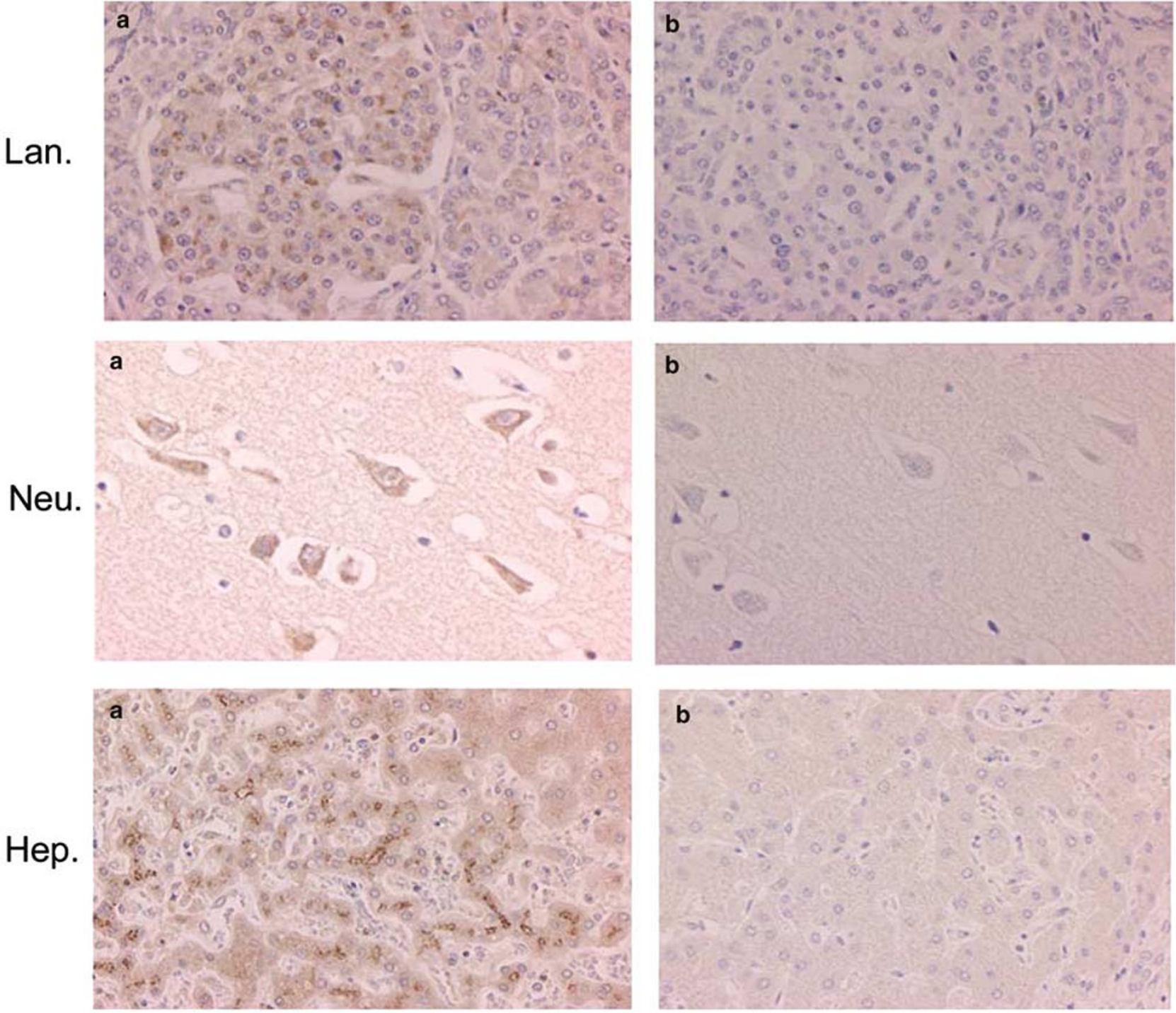

Figure 9 Absorption test. Immunostaining with the esRAGE-specific antibody without absorption (a) and with preabsorption (b). All esRAGE-positive staining completely disappeared after absorption. Lan., Langerhans' islet; Neu., neurons; Hep., hepatocytes.

insulin immunoreactivity was marked on an electron microgram (data not shown). Neurons of the cerebellum and cerebrum, and medullary cells of the adrenal gland also expressed esRAGE with pattern A. These results indicate that esRAGE expressed with this pattern may be associated with neuroendocrine activity. HMGB-1 (also known as amphoterin), an endogenous RAGE ligand, was shown to promote the RAGE-dependent neuronal differentiation. ${ }^{7,8}$ 

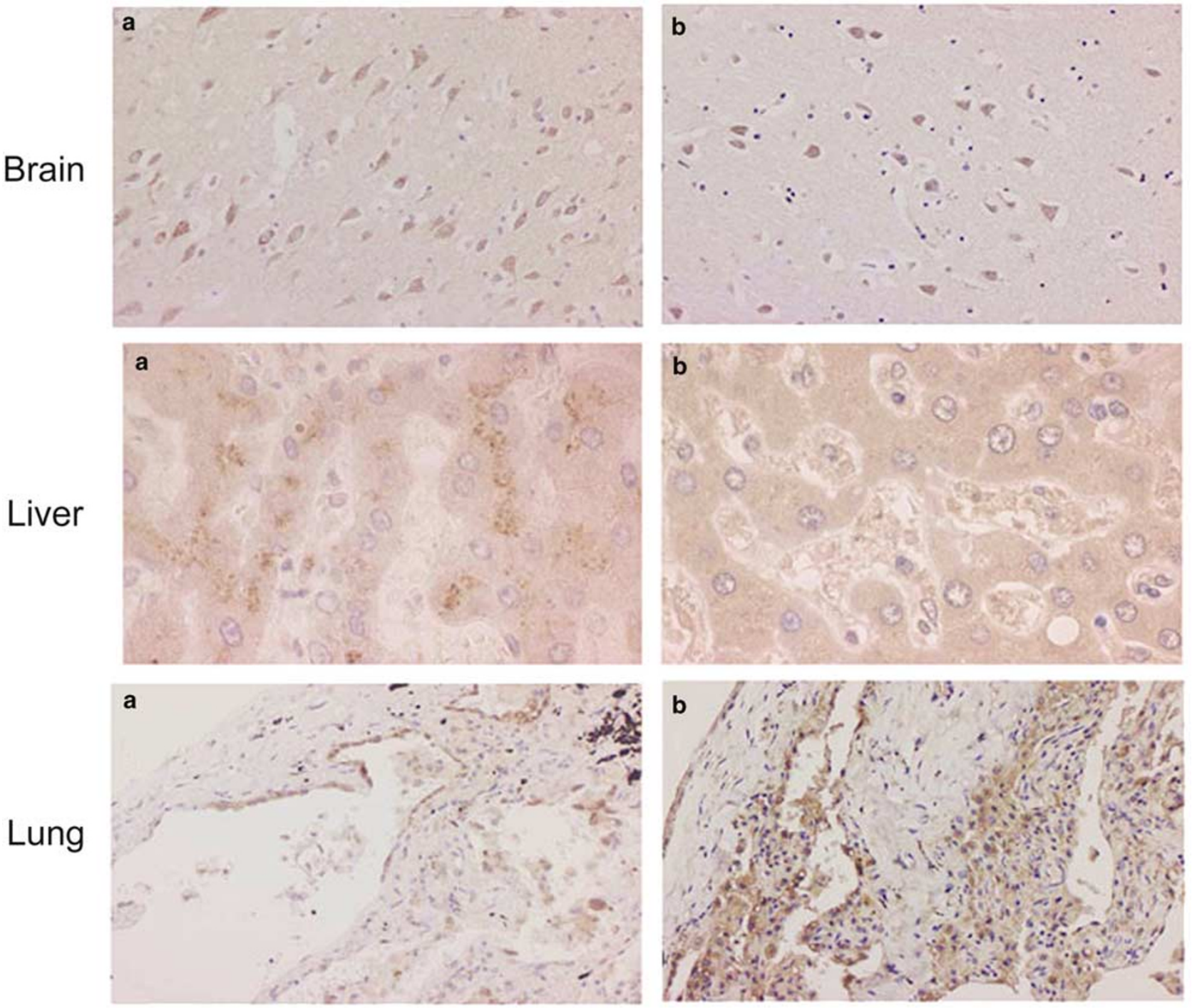

Figure 10 Comparison of the expression patterns obtained with the antibodies against esRAGE and the V-type domain of RAGE. Immunostaining with the antibody against esRAGE (a) or against V-type domain of RAGE (b) is shown in the brain, liver, and lung. A similar immunostaining pattern was observed with each antibody in almost all samples we investigated, such as the brain and lung. A distinct different pattern was observed in hepatocytes with dot-like cytoplasmic staining by an esRAGE-specific antibody and diffuse cytoplasmic staining by the antibody against the V-type domain of RAGE.

The esRAGE mRNA was initially isolated from primary cultured human EC. ${ }^{3}$ In this study, we detected esRAGE protein in vascular EC with pattern A.

Schraml et al reported that two cDNA sequences coded for pulmonary surfactant-associated protein B and RAGE have homology to genes specifically expressed in the lung. ${ }^{9}$ The diffuse cytoplasmic expression of esRAGE in type II pneumocytes, which synthesize and secrete surfactants, may be related to the functional maturation of this cell type. esRAGE expression with pattern A on macrophages and mononuclear cells was also noteworthy. Extracellular newly identified RAGE-binding protein (EN-RAGE) and related members of the S100/ calgranulin superfamily are reportedly released by inflammatory cells targeted to inflammation sites. ${ }^{10-14}$ This result may support the assumption that esRAGE is a molecular device protecting against these inflammatory endogenous RAGE-binding proteins.

Pattern B appears to be related to exocrine functions. Cells belonging to this category were exemplified by the epithelia of bile ducts, hepatocytes, glandular epithelia of the pancreas, acinus and ductal epithelia of the salivary gland, follicular epithelia of the thyroid, glandular epithelia of the bronchiole, superficial-foveolar epithelia and glands of the gastrointestinal tract, renal tubular epithelia, glandular epithelia of the prostate, and ductal epithelia of the accessory gland of the skin. Double staining with antibodies against esRAGE and 

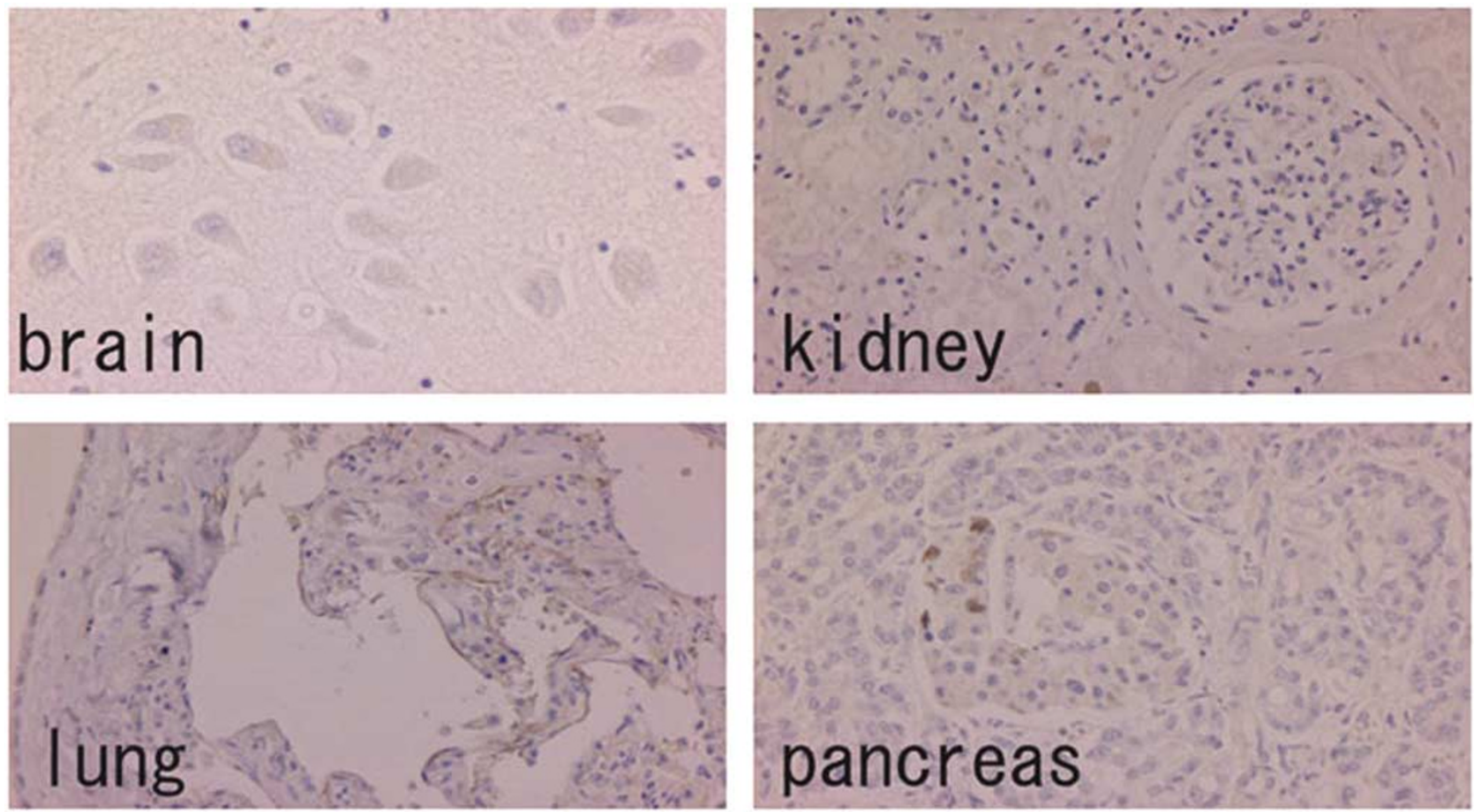

Figure 11 Positive immunostaining with the antibody against the carboxy-terminus of RAGE was restricted to a few specimens, including some regenerated type II pneumocytes (lung) and several cells of Langerhans' islet (pancreas). In other cases examined, positive staining was not detected. Two figures (brain and kidney) are shown as negative staining.

Table 1 The immunostaining patterns of esRAGE in the main human organs investigated

Characteristic expressed Loci distributed

\section{A pattern \\ Diffuse cytoplasmic staining}

\section{B pattern \\ Dot-like supranuclear granular expression facing the luminal surface}

\section{$C$ pattern}

Diffuse staining in the stromal area
D pattern
Diffuse and strong staining in the secreted materials

\author{
Neuron cell body and dendrite of the cerebellum and cerebrum \\ Vascular EC \\ Pneumocytes \\ Mesothelial cells \\ $\beta$ cells of pancreatic islet; acinus of the pancreas \\ Medullary cells of the adrenal gland \\ Macrophages and mononuclear phagocytes
}

\section{Hepatocytes}

Epithelia of bile ducts

Acinus of the salivary gland

Basal cells of the squamous epithelia of the esophagus

Surface and foveolar epithelia and gland of the digestive tract

Renal tubules

Prostatic gland

Basal cells of epidermis and ductal epithelia of the accessory gland of the skin Follicular epithelia of the thyroid

Epithelia of the bronchiole

The wall of the artery (coronary, aorta)

Colloid of the thyroid

Crystals in the renal tubular lumen

Secretion products in prostatic gland
CD10 has demonstrated that esRAGE is localized along the lines of bile canaliculi in the hepatocytes. esRAGE expression decreased in hepatocytes of patients with obstructive jaundice and increased in the ductal epithelia of the salivary glands of patients with Sjogren's syndrome (data not shown). The 
Table 2 esRAGE expression with a distinct pattern in individual organs

\section{Brain}

Thyroid

Salivary gland

Heart

Artery

Lung

Bronchi

Liver

Pancreas

Kidney

Adrenal gland

Esophagus

Digestive tract

Prostate

Skin
Neuron cell body and dendrite of the cerebellum and cerebrum with A pattern staining

Follicular epithelia with weak B pattern staining; colloid with D pattern staining

Acinus and glandular epithelia with B pattern staining

Mesothelia and cardiac muscle with A pattern staining

EC with A pattern staining; stroma of wall with C pattern staining

Pneumocytes and macrophages and mononuclear phagocytes in alveolar lumen with A pattern staining Epithelia with weak B pattern staining

Hepatocytes and epithelia of the bile duct with stronger B pattern staining

$\beta$ cells of pancreatic islet with A pattern staining; acinus with weak A pattern staining

Renal tubular epithelia with B pattern staining; crystal in the glandular lumen with D pattern

Medullary cells with A pattern staining

Basal cells of the squamous epithelia with B pattern staining

Surface and foveolar epithelia and gland with B pattern staining

Glandular epithelia with B pattern staining; secretion in the glandular lumen with D pattern staining

Basal cells of the epidermis and ductal epithelia of the accessory gland with B pattern staining results suggest that esRAGE may contribute to secretion of bile and saliva and/or their absorption.

In pattern C, esRAGE was found to colocalize with HSPG in the same stromal area of the aorta and coronary artery of individuals with mild atherosclerosis, indicating that the storage of esRAGE in the extracellular matrix may depend on HSPG. As HSPG is a component of fresh fibrosis, esRAGE may be associated with the extension of fibrosis as a consequence of inflammation. ${ }^{15-18}$

When the tissues were probed with goat polyclonal antibody against V-type region of RAGE, the resultant staining was very similar to that with rabbit polyclonal antibody specific to esRAGE (Figure 10). On the other hand, when the tissues were probed with rabbit polyclonal antibody against the carboxy-terminal intracellular domain of RAGE, staining was restricted and weak (Figure 11). As the former antibody recognizes full-length RAGE and esRAGE, and as the third one recognizes full-length and N-terminally truncated RAGE but not esRAGE, as shown in Figure 1, these observations led to an interpretation that esRAGE may be a predominant form of RAGE proteins, or that the secretory variant may be stable in intra- and extracellular milieux, having longer half-lives than the other forms.

RAGE is known as a multiligand receptor for AGE, ${ }^{19,20}$ amyloid $\beta$ proteins, ${ }^{21}$ S100/calgranulins, ${ }^{10}$ HMGB- $1,{ }^{22,23}$ and $\beta 2$-integrin Mac- $1{ }^{24}$ Binding of these ligands to RAGE initiates sustained cellular activation mediated by receptor-dependent signaling involving the transcription factor NF- $\kappa \mathrm{B}^{25}$ Our data suggested that the actions of RAGE in both physiological and pathological processes are mediated through complex interplay between the RAGE isoforms, and are not mediated solely by the full-length RAGE. In particular, determination of the level or the ratio of individual RAGE isoforms may predict the susceptibility to various disease conditions. We have recently developed an ELISA system for esRAGE, and applied it to serum samples from type I diabetic subjects. Blood esRAGE levels significantly declined as the retinal status advanced to diabetic retinopathy (unpublished data). esRAGE seemed to confer protection against diabetic retinopathy, an earlier onset complication. In future studies, it will be important to identify the molecular mechanisms responsible for the alternative splicing of RAGE.

In conclusion, this is the first study that revealed the distribution of esRAGE in normal human adult tissues and organs with RAGE domain-specific antibodies. This study will contribute to understanding and discussing the physiological and pathological basis of esRAGE in humans. We hope this study will open new avenues in providing a novel diagnostic approach and therapeutic concept in RAGE- or esRAGE-related diseases.

\section{Acknowledgements}

We thank Professor Takashi Saka of Niigata University for the gift of anti-HSPG antibody. In addition, we particularly thank Tokimasa Kumada and Hideki Hatta of the First Pathology Department of Toyama Medical and Pharmaceutical University for their excellent technical support. This work was supported in part by a grant from the 21st century COE program in Japan, R\&D, for the practical use of university-based technology, by matching government funds and private funds from the Ministry of Economy, Trade and Industry of Japan (No. 0320003), grants-in-aid for scientific research from the Japan Society for the Promotion of Science (No. 16790183), and from the Honjin and Japan Diabetes Foundations.

\section{References}

1 Schmidt AM, Yan SD, Yan SF, et al. The multiligand receptor RAGE as a progression factor amplifying 
immune and inflammatory responses. J Clin Invest 2001;108:949-955.

2 Neeper M, Schmidt AM, Brett J, et al. Cloning and expression of a cell surface receptor for advanced glycosylation end products of proteins. J Biol Chem 1992;267:14998-15004.

3 Yonekura H, Yamamoto Y, Sakurai S, et al. Novel splice variants of the receptor for advanced glycation end-products expressed in human vascular endothelial cells and pericytes, and their putative roles in diabetesinduced vascular injury. Biochem J 2003;370:10971109.

4 Fejzo MS, Slamon DJ. Frozen tumor tissue microarray technology for analysis of tumor RNA, DNA, and proteins. Am J Pathol 2001;159:1645-1650.

5 Kumada T, Tsuneyama K, Hatta H, et al. Improved onehour rapid immunostaining method using intermittent microwave irradiation: practicability based on five years application in Toyama Medical and Pharmaceutical University Hospital. Mod Pathol 2004;17:11411149.

6 Tsuneyama K, Harada K, Yasoshima M, et al. Monocyte chemotactic protein-1, -2 , and -3 are distinctively expressed in portal tracts and granulomata in primary biliary cirrhosis: implications for pathogenesis. J Pathol 2001;193:102-109.

7 Hori O, Brett J, Slattery T, et al. The receptor for advanced glycation end products (RAGE) is a cellular binding site for amphoterin. Mediation of neurite outgrowth and co-expression of rage and amphoterin in the developing nervous system. J Biol Chem 1995; 270:25752-25761.

8 Huttunen HJ, Kuja-Panula J, Rauvala H. Receptor for advanced glycation end products (RAGE) signaling induces CREB-dependent chromogranin expression during neuronal differentiation. J Biol Chem 2002;277: 38635-38646

9 Schraml P, Shipman R, Colombi M, et al. Identification of genes differentially expressed in normal lung and non-small cell lung carcinoma tissue. Cancer Res 1994;54:5236-5240.

10 Stern D, Yan SD, Yan SF, et al. Receptor for advanced glycation endproducts: a multiligand receptor magnifying cell stress in diverse pathologic settings. Adv Drug Deliv Rev 2002;54:1615-1625.

11 Hofmann MA, Drury S, Fu C, et al. RAGE mediates a novel proinflammatory axis: a central cell surface receptor for S100/calgranulin polypeptides. Cell 1999; 97:889-901.
12 Schafer BW, Heizmann CW. The S100 family of EFhand calcium-binding proteins: functions and pathology. Trends Biochem Sci 1996;21:134-140.

13 Donato R. Functional roles of S100 proteins, calciumbinding proteins of the EF-hand type. Biochim Biophys Acta 1999;1450:191-231.

14 Kerkhoff C, Klempt M, Sorg C. Novel insights into structure and function of MRP8 (S100A8) and MRP14 (S100A9). Biochim Biophys Acta 1998;1448:200-211.

15 Thompson HL, Burbelo PD, Gabriel G, et al. Murine mast cells synthesize basement membrane components. A potential role in early fibrosis. J Clin Invest 1991;87:619-623.

16 McGrath JA, Eady RA. Heparan sulphate proteoglycan and wound healing in skin. J Pathol 1997;183:251-252.

17 Maruyama S, Cantu III E, DeMartino C, et al. Interaction of baboon anti-alpha-galactosyl antibody with pig tissues. Am J Pathol 1999;155:1635-1649.

18 Venkatesan N, Ebihara T, Roughley PJ, et al. Alterations in large and small proteoglycans in bleomycininduced pulmonary fibrosis in rats. Am J Respir Crit Care Med 2000;161:2066-2073.

19 Yamamoto Y, Kato I, Doi T, et al. Development and prevention of advanced diabetic nephropathy in RAGE-overexpressing mice. J Clin Invest 2001;108: 261-268.

20 Park L, Raman KG, Lee KJ, et al. Suppression of accelerated diabetic atherosclerosis by the soluble receptor for advanced glycation endproducts. Nat Med 1998;4:1025-1031.

21 Yan SD, Chen X, Fu J, et al. RAGE and amyloid-beta peptide neurotoxicity in Alzheimer's disease. Nature 1996;382:685-691.

22 Rouhiainen A, Kuja-Panula J, Wilkman E, et al. Regulation of monocyte migration by amphoterin (HMGB1). Blood 2004;104:1174-1182.

23 Taguchi A, Blood DC, del Toro G, et al. Blockade of RAGE-amphoterin signalling suppresses tumour growth and metastases. Nature 2000;405:354-360.

24 Chavakis T, Bierhaus A, Al-Fakhri N, et al. The pattern recognition receptor (RAGE) is a counterreceptor for leukocyte integrins: a novel pathway for inflammatory cell recruitment. J Exp Med 2003;198:1507-1515.

25 Huttunen HJ, Fages C, Rauvala H. Receptor for advanced glycation end products (RAGE)-mediated neurite outgrowth and activation of NF-kappaB require the cytoplasmic domain of the receptor but different downstream signaling pathways. J Biol Chem 1999; 274:19919-19924. 